\title{
On the equivalence of pairing correlations and intrinsic vortical currents in rotating nuclei
}

\author{
H. Laftchiev ${ }^{1,2}$, D. Samsœn ${ }^{1}$ P. Quentin ${ }^{1,3}$, and I. N. Mikhailov ${ }^{4,5}$ \\ ${ }^{1}$ Centre d'Études Nucléaires de Bordeaux-Gradignan (CNRS-IN2P3 and Université Bordeaux I), Le Haut Vigneau BP 120, \\ F-33175 Gradignan, France \\ ${ }^{2}$ Institute of Nuclear Research and Nuclear Energy (Bulgarian Academy of Sciences), Tzarigradsko Chaussee 72, 1784 Sofia, \\ Bulgaria \\ ${ }^{3}$ Theoretical Division, T-DO (Los Alamos National Laboratory), PO Box 1663, Los Alamos, NM 87545, USA \\ ${ }^{4}$ Bogoliubov Laboratory of Theoretical Physics (Joint Institute of Nuclear Research), Joliot-Curie str. 6, 141980 Dubna \\ (Moscow Region), Russia \\ ${ }^{5}$ Centre de spectrométrie Nucléaire et de Spectrométrie de Masse, (CNRS-IN2P3 and Université Paris XI), Bât. 104, F-91406 \\ Orsay-Campus, France
}

\begin{abstract}
The present paper establishes a link between pairing correlations in rotating nuclei and collective vortical modes in the intrinsic frame. We show that the latter can be embodied by a simple Stype coupling à la Chandrasekhar between rotational and intrinsic vortical collective modes. This results from a comparison between the solutions of microscopic calculations within the HFB and the HF Routhian formalisms. The HF Routhian solutions are constrained to have the same Kelvin circulation expectation value as the HFB ones. It is shown in several mass regions, pairing regimes, and for various spin values that this procedure yields moments of inertia, angular velocities, and current distributions which are very similar within both formalisms. We finally present perspectives for further studies.
\end{abstract}

\section{INTRODUCTION AND METHODS}

In a recent paper [1], some of the authors of the present article conjectured that the well-known dynamical effects of pairing correlations in rotating nuclei, explicited in the significant decrease of the moments of inertia from rigid body values $[2,3]$, could be pictured as a vortical intrinsic collective motion coupled to the global rotation of the socalled intrinsic (body-fixed) frame relatively to the laboratory system. Namely, one intuitively would think of such a mode as aligned and counter-rotating with respect to the global angular velocity so as to produce the above quoted reduction of the moments of inertia. Furthermore, if one wants to preserve the nuclear shape in the presence of this intrinsic vortical mode the latter should be tangential to the grossly-defined nuclear surface.

As often suggested after R. Y. Cusson [4] (see also Ref. [5] for some relevant references), a simple ansatz for such a coupling is provided by the S-ellipsoid fluid dynamics as studied classically in great details by Chandrasekhar in the context of celestial self-gravitating objects [6]. It makes use of a velocity field which is linear in the coordinates and produces a coupling of a global rotation with an intrinsic mode corresponding to a motion tangential to the body surface which is supposed to be ellipsoidal. Furthermore, the vorticities (i.e., the curl) of the two velocity fields are assumed to be aligned or anti-aligned along a principal axis of the ellipsoid. As shown, e.g., in Ref. [7] (see Fig. 1 there) such a simple parameterization is capable of yielding a variety of modes in the laboratory frame from rigid-body rotation to irrotational modes as well as various shear modes.
The connection of this S-ellipsoid fluid dynamics with pairing correlations may be discussed at various levels. First, one may note that a tangential intrinsic mode which is not changing the body shape (in the $\vec{r}$-sector of the phase-space) is only able to redistribute the density function in the $\vec{p}$-sector of the phase-space. Clearly, up to self-consistent couplings between the momentum redistribution and the body shape, the BCS pairing correlations behave exactly in the same way (redistributing the single-particle momenta). Second, in the semiclassical (Thomas-Fermi) calculations of Ref. [8], upon widely varying the pairing strength in some deformed rare-earth rotating nuclei, the authors have unambiguously exhibited current patterns very similar indeed to the classical current patterns of S-ellipsoids. Such currents had been shown before to exist in paired solutions at finite spin $[9,10]$. They were however embedded in the midst of shell-effect-generated intrinsic currents whose existence had been found long ago (see the non-paired rotating solutions of [11]) and which will be further discussed in Section II A below.

However, the arguments above sketched are purely qualitative. It is the aim of this paper to investigate how quantitatively correct they might be.

To achieve this goal, it is first necessary to find a tool to quantify the intrinsic vortical content of a given current distribution. Within the context of S-ellipsoid fluids, it is rather natural to use the Kelvin circulation whose components are defined by (see, e.g., [12])

$$
\hat{K}_{k}=\sum_{i, j} \epsilon_{i j k}\left(\frac{a_{j}}{a_{i}} x_{i} p_{j}-\frac{a_{i}}{a_{j}} x_{j} p_{i}\right)
$$


where $\epsilon_{i j k}$ is the totally antisymmetrical third rank tensor and the $a_{i}$ 's are characteristic lengths associated with the ellipsoidal shape of the nuclei (e.g., the semi-axes). These lengths are proportional to $\sqrt{\left\langle x_{i}^{2}\right\rangle}$. These operators clearly appear as the components of a doublystretched (in both $\vec{r}$ and $\vec{p}$ ) orbital angular momentum. Physically, they correspond to a rotation in the intrinsic frame of a sphere obtained by stretching the ellipsoidal intrinsic distribution. Indeed as shown by Lebowitz (see, e.g., Ref. [6]), the S-ellipsoid coupling mode may be understood as a global rotation along some principal axis $\vec{u}$, followed by the stretching to a sphere and the rotation (according to the same axis $\vec{u}$ of the considered ellipsoid) as above described, and finally by the inverse stretching back to the original ellipsoidal shape.

To check the validity of the above mentioned conjecture, we compare the results of two types of microscopic calculations, one involving pairing correlations in a purely rotating formalism, the other with no pairing but imposing the S-ellipsoid dynamics for the global rotation and intrinsic vortical mode velocity fields as well as for their coupling.

The pairing correlations are treated à la Hartree-FockBogoliubov (HFB) using a Skyrme effective interaction in the particle-hole channel and a seniority force in the pairing channel within a code developed by $\mathrm{H}$. Laftchiev $[13,14]$. The triaxial character of the solutions arising from the time-reversal symmetry breaking inherent to rotating nuclei is treated within the Fourier decomposition formalism described in Ref. [15]. Namely, the wavefunctions and relevant densities are defined in cylindrical coordinates on a mesh in the two spatial directions $(r, z)$ and as Fourier series in the azimuthal angle $\theta$. An approximate description of particle-number projection within the Lipkin-Nogami (LN) scheme [16] is also available in the code.

The S-ellipsoid coupling case is described within the Hartree-Fock-Skyrme generalized Routhian formalism of Refs. $[15,17]$. This formalism makes it possible to describe the S-ellipsoid dynamics upon using a double constraint on both angular momentum and Kelvin circulation via two angular velocities $\Omega$ and $\omega$ (relevant to each collective mode) along the first-axis.

These two microscopic approaches have been used in the following scheme. In a first step, we have used the HFB formalism with a constraint on angular momentum using realistic pairing strengths. We thus generate vortical currents in the intrinsic frame which can be quantified for instance by the mean value of the Kelvin circulation operator measured in the intrinsic frame and defined as

$$
\left\langle\hat{K}_{1}\right\rangle=\int(\tilde{\vec{r}} \times \tilde{\vec{j}})_{1} \mathrm{~d}^{3} r .
$$

There $\tilde{\vec{r}}$ is the stretched position vector and $\tilde{\vec{j}}$ is the stretched current in the intrinsic frame whose components are obtained after subtracting the rotating-part to the usual current-density $\vec{j}$ as
TABLE I. Pairing strength $G_{n}$ and $G_{p}$ for neutrons and protons as well as energy cutoff $\mu$ (in $\mathrm{MeV}$ ) for each of the three studied nuclei. The matrix elements $g_{q}$ of the seniority force are defined by the usual prescription $g_{q}=G_{q} /\left(11+N_{q}\right)$. The configuration space includes all single-particle states whose energies lies below $\epsilon+\mu$ where $\epsilon$ is the chemical potential.

\begin{tabular}{lccc}
\hline \hline & ${ }^{150} \mathrm{Gd}$ & ${ }^{192} \mathrm{Hg}$ & ${ }^{254} \mathrm{No}$ \\
\hline$G_{n}$ & 14.4 & 11.5 & 13.8 \\
$G_{p}$ & 14.4 & 11.5 & 15.0 \\
Cutoff $(\mu)$ & 5 & 10 & 7 \\
\hline \hline
\end{tabular}

$$
\tilde{j}_{i}=a_{i}\left[\vec{j}-\frac{m}{\hbar}(\vec{\Omega} \times \vec{r}) \rho\right]_{i} .
$$

where $\vec{j}$ is the usual quantum mechanical current density defined as

$$
\vec{j}=\left.\frac{1}{2 i}\left(\vec{\nabla}_{\vec{r}^{\prime}}-\vec{\nabla}_{\vec{r}}\right) \rho\left(\vec{r}, \vec{r}^{\prime}\right)\right|_{\vec{r}=\vec{r}^{\prime}}
$$

The mean value $I$ of the angular momentum operator is defined through

$$
\sqrt{I(I+1)}=\langle\hat{I}\rangle=\int(\vec{r} \times \vec{j}+\vec{\rho})_{1} \mathrm{~d}^{3} r
$$

where $\vec{\rho}$ is the spin-vector density.

In a second step, we perform pure Hartree-Fock calculations (i.e., without pairing) with a double constraint on both angular momentum and Kelvin circulation to obtain the same values $I$ and $\left\langle\hat{K}_{1}\right\rangle$ as in the preceding step. In the following, this type of calculations will be referred to as $\mathrm{HF}+\mathrm{V}$ calculations.

The paper will be organized as follows. In the next Section we will present and compare the results of the numerical calculations yielded by the two formalisms, namely $\mathrm{HFB}$ and $\mathrm{HF}+\mathrm{V}$ in several mass regions. Section III will be devoted to a summary of the salient features exhibited in Section II and to some perspectives for further studies.

\section{RESULTS}

Numerical calculations have been performed for three heavy nuclei: two in the $A \sim 150$ and $A \sim 190$ superdeformation regions (namely for the ${ }^{150} \mathrm{Gd}$ and the ${ }^{192} \mathrm{Hg}$ nuclei) including LN approximate particle-number projection scheme and one very heavy nucleus $\left({ }^{254} \mathrm{No}\right)$ without LN projection. The $\mathrm{SkM}^{*}$ parameterization [18] of the Skyrme effective force has been used. The pairing strengths $G_{q}$ for the charge state $q$ are given for each calculated nuclei in table I together with the corresponding single-particle configuration space in use. For all three nuclei, kinetic $\left(\mathfrak{J}^{(1)}\right)$ and dynamical $\left(\mathfrak{J}^{(2)}\right)$ moments of inertia are calculated using the formulae 

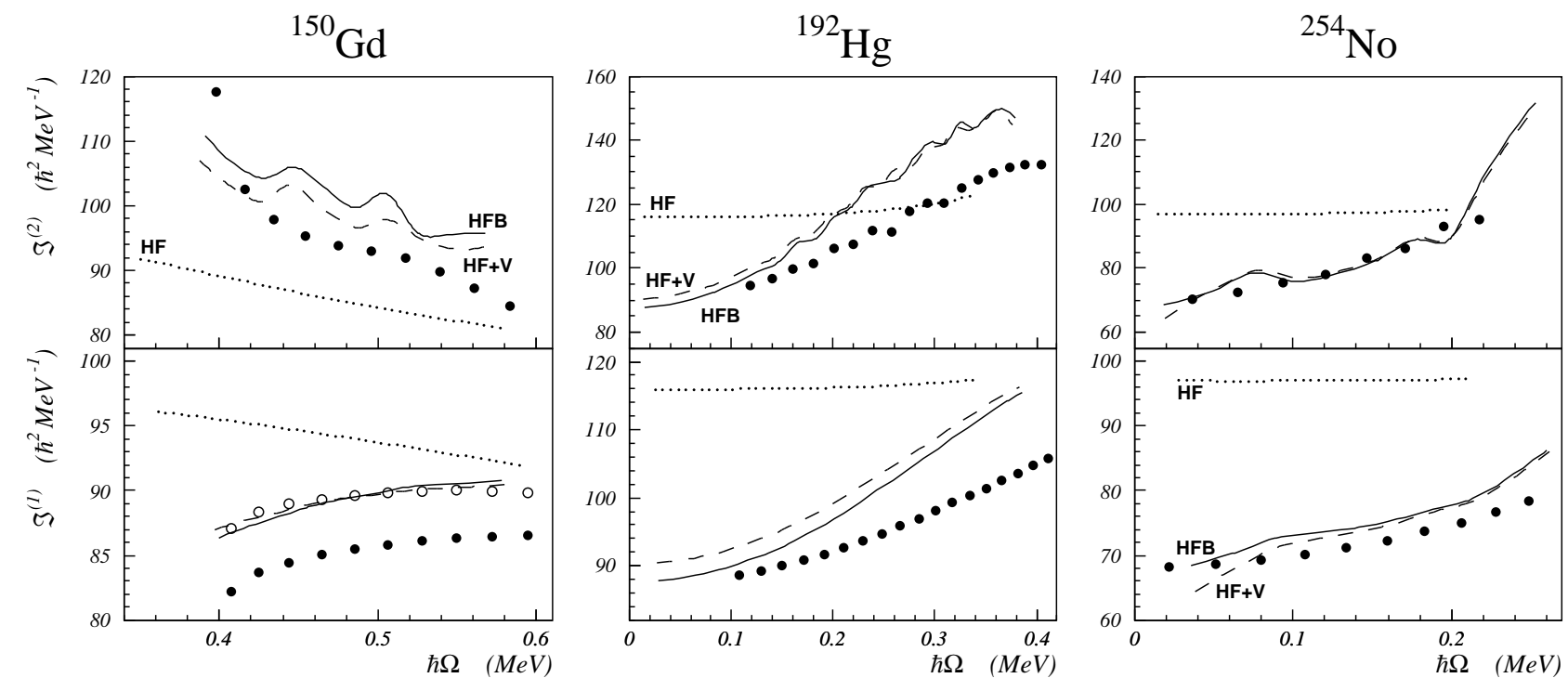

FIG. 1. Dynamical (upper panel) and kinetic (lower panel) moments of inertia (in $\hbar^{2} \mathrm{MeV}^{-1}$ ) for the three considered nuclei as functions of the angular velocity $\Omega$ in $\mathrm{MeV}$. The conventions in use are the following: HF value (dotted line), HFB value (full line), and $\mathrm{HF}+\mathrm{V}$ value (dashed line). Experimental data are represented as full circles, excepted for the ${ }^{150} \mathrm{Gd}$ kinetic moment of inertia with the assumption $I_{\text {init }}=34 \hbar$ as opened circles (lower left panel). The Lipkin-Nogami correction has been applied for ${ }^{150} \mathrm{Gd}$ and ${ }^{192} \mathrm{Hg}$.

$$
\mathfrak{J}^{(1)}=\frac{\langle\hat{I}\rangle}{\Omega},
$$

and

$$
\mathfrak{J}^{(2)}=\frac{\partial\langle\hat{I}\rangle}{\partial \Omega}
$$

As was already mentioned in a previous paper [14], using these expressions for the moments of inertia, we assume that they can be computed as well from the energycorrected LN wavefunctions. We also assume that the angular velocity dependence of the estimate of $\mathrm{LN} \lambda_{2}$ parameter is small, otherwise $\mathfrak{J}^{(2)}$ would have to be evaluated through second derivatives of the energy with respect to $\Omega$.

\section{A. The yrast superdeformed band of ${ }^{150} \mathrm{Gd}$}

Up to now, fourteen superdeformed bands have been experimentally found in ${ }^{150} \mathrm{Gd}[19,20]$. We will deal here with the yrast superdeformed band only. Using the Cranked-HF approach, it was impossible [15] to reproduce the trends of the experimental kinetic and dynamical moments of inertia of this band as can be seen in Fig. 1 (dotted line).

The results presented in the upper panel of Fig. 1 for the dynamical moment of inertia of this nucleus show a much better agreement with experimental data within the $\mathrm{HFB}+\mathrm{LN}$ formalism as compared to the HF one. Indeed, we now reproduce the decrease of this moment of inertia along the band with theoretical values which are rather close to the experimental ones as was previously shown within pure HFB calculations by Bonche et al. [21]. In the lower panel, the agreement of HFB+LN kinetic moment of inertia with the experimental data of Ref. [19], though much better than the HF one, is seen to be rather poor. However, the experimental value of this moment of inertia relies on an assumption of the lowest spin value of the observed band [see eq. (6)] which has been assumed in [19] to be $I_{\text {init }}=32 \hbar$. Taking now for the initial spin of the band $I_{\text {init }}=34 \hbar$ shifts up the experimental data and then yields a very good agreement with $\mathrm{HFB}+\mathrm{LN}$ values of the moment of inertia.

Let us now focus on the intrinsic vortical content of the current density. In the upper part of Fig. 2 the Kelvin circulation mean value is plotted as a function of the angular velocity for ${ }^{150} \mathrm{Gd}$. One may first notice that the Kelvin circulation mean value in the HF case is not zero, even though the intrinsic vorticity constraint is absent. It is negative (with a rather small absolute value of about $1 \hbar$ ) for the protons while it is positive and larger (about $2 \hbar$ ) for the neutrons. These contributions should most likely be attributed to small inhomogeneities in the density due to shell effects. It is almost constant for the neutrons and slightly decreasing with $\Omega$ for the protons. Dubbing this current patterns as shell effects may also be understood in rather general terms. In Ref. [22] within purely semiclassical microscopic calculations, it has been shown that for finite systems small surface-peaked counter-rotating intrinsic currents do appear. This effect is analogous to the diamagnetism which is observed in an electronic system submitted to a constant magnetic field. Thence the 

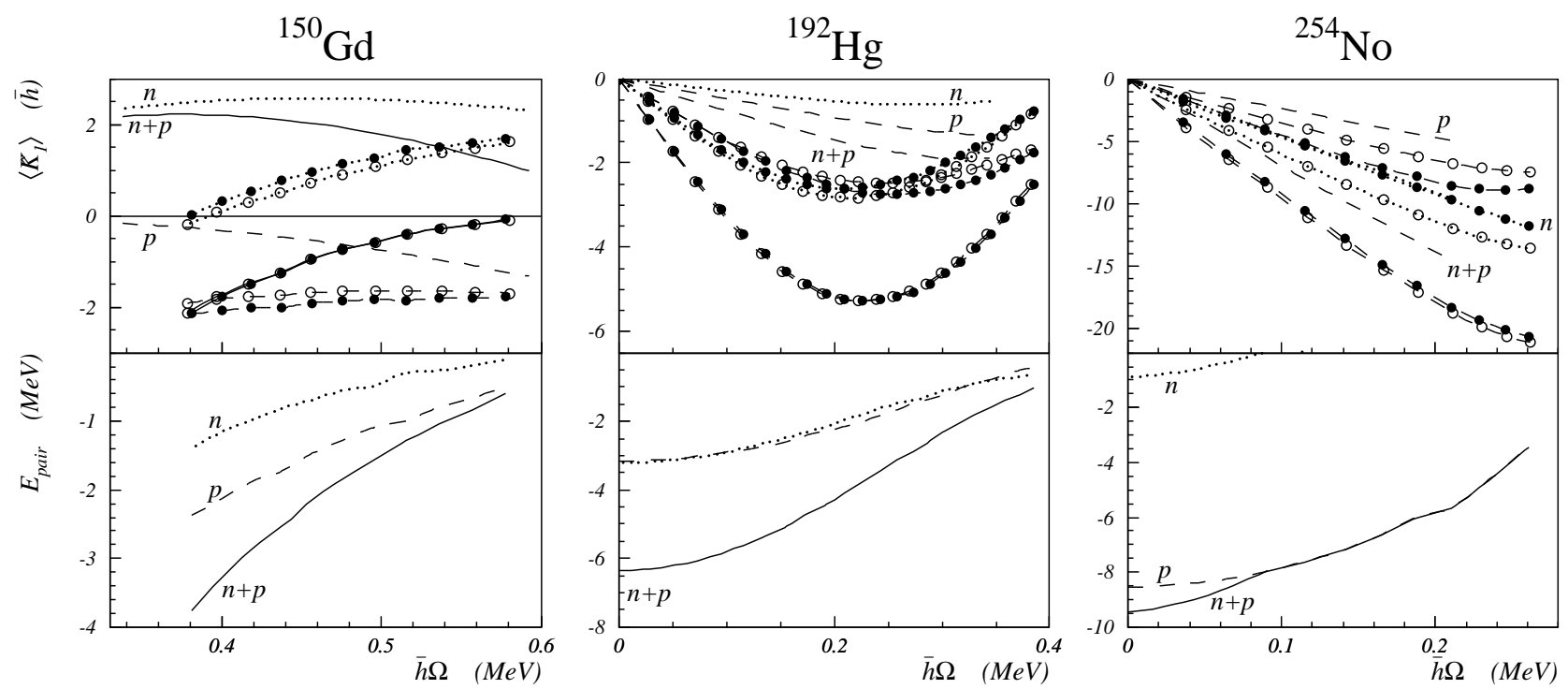

FIG. 2. Kelvin circulation mean value in $\hbar$ units (upper panel) and pairing energy in MeV (lower panel) for the three considered nuclei as functions of the angular velocity $\Omega$ in $\mathrm{MeV}$. The conventions in use are the following: proton contributions (dashed line), neutron contributions (dotted line), and total contributions (full line); in the upper panel: HF value (no symbols), $\mathrm{HF}+\mathrm{V}$ value (opened circles), $\mathrm{HFB}$ value (full circles). The Lipkin-Nogami correction has been applied for ${ }^{150} \mathrm{Gd}$ and ${ }^{192} \mathrm{Hg}$.

origin of all intrinsic currents (in Routhian HF calculations) which are not surface-peaked and counter-rotating (as obtained years ago in, e.g., [11]) are clearly and properly to be attributed to shell effects.

Comparing now the HF curves with the HFB ones, we see that the introduction of pairing correlations shifts down the Kelvin circulation mean value by about $2 \hbar$ for each charge state at low angular velocities and still $0.5 \hbar$ units at the end of the band. This decrease of the Kelvin circulation in the HFB case is related, as it has been already demonstrated in [1], to the fact that the collective effect of pairing correlations is equivalent to a counterrotating intrinsic vortical motion (see also Ref. [8]). The combination of the above mentioned HF variation of $\left\langle\hat{K}_{1}\right\rangle$ with $\Omega$ for protons and neutrons together with the regular decrease of the pairing correction as described previously yields an increase of the neutron contribution and an almost constant pattern for the protons. As a result the total Kelvin circulation exhibits an $\Omega$ variation similar to the neutron one.

This decreasing pairing effect on the ${ }^{150} \mathrm{Gd}$ Kelvin circulation is nicely correlated, as can be seen on the lower panel of Fig. 2, with the decrease of the pairing energy (defined as a trace of the product of the abnormal density with the gap potential). The latter is a well-known behavior, known as the Mottelson-Valatin effect [23], similar to the effect of a magnetic field on a superconductor below the critical point [24]. Indeed as the pairing energy almost vanishes at high angular velocity, the Kelvin circulation in the HFB formalism almost reaches its HF value.

As said in the Introduction, the Kelvin circulation is a powerful tool to measure the intrinsic vortical content of the current distribution. In Fig. $3\left({ }^{150} \mathrm{Gd}\right.$ bottom part) we present the current patterns in the intrinsic frame of two rotational states of the HFB superdeformed yrast band of ${ }^{150} \mathrm{Gd}$, namely for $I=32 \hbar$ and $I=46 \hbar$ (corresponding to angular velocities $\Omega=381 \mathrm{keV}$ and $\Omega=516 \mathrm{keV})$. In both cases, the neutron current patterns are rather disordered (due to shell structure effects). This is related to positive values of the neutron contribution to the Kelvin circulations. On the contrary, proton current patterns are rather well oriented, at least around the nuclear surface, along elliptic lines parallel to the surface, the more so in the $I=32 \hbar$ case where total Kelvin circulation and pairing energies are greater (in absolute value) than for $I=46 \hbar$. This trend is due to the fact (observed in Fig. 2) that the proton HF contribution to $\left|\left\langle\hat{K}_{1}\right\rangle\right|$ is very small. In the HF case (upper part), the current patterns show no particular order for both spins, and for protons as well as for neutrons. This clearly shows that the inclusion of pairing correlations favors the appearance of S-ellipsoid-like currents in rotating nuclei.

We then have performed $\mathrm{HF}+\mathrm{V}$ calculations, namely constraining HF solutions to have $I$ and $\left\langle\hat{K}_{1}\right\rangle$ values [see eqs. (2) and (5)] identical to their HFB counterparts. The constraint in use is of an isoscalar type, that is constraining on the total (neutron + proton) angular momentum and Kelvin circulation. However, it can be seen in Fig. 2 that the proton and neutron contributions to the Kelvin circulation in $\mathrm{HF}+\mathrm{V}$ calculations are very close to what is obtained in HFB calculations even though these contributions have not been constrained separately. Moreover, the current patterns for the $\mathrm{HFB}$ and $\mathrm{HF}+\mathrm{V}$ approaches (see Fig. 3) are similar. Hence we have successfully grafted the superfluid part of the HFB currents 


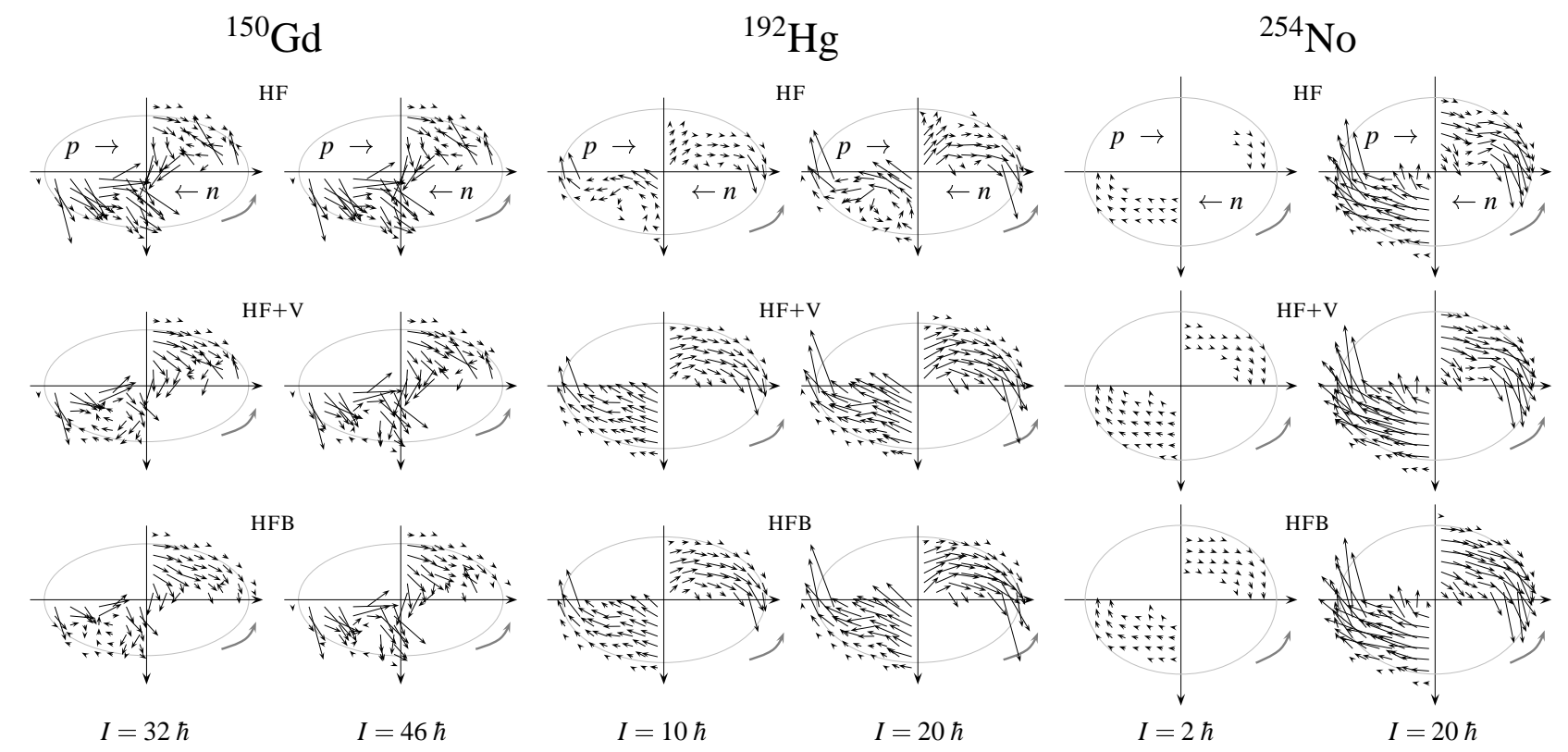

FIG. 3. Current patterns in the intrinsic frame for two rotational states in the three considered nuclei. For each nucleus. The same arbitrary units for the length of $|\vec{j}|$ are used for both spins. In all ellipses, the proton (resp. neutron) current patterns are represented in the top-right (resp. bottom-left) quarter. The global rotation direction is counter-clockwise. The formalisms used are $\mathrm{HF}, \mathrm{HF}+\mathrm{V}$ and $\mathrm{HFB}$ from top to bottom. The Lipkin-Nogami correction has been applied for ${ }^{150} \mathrm{Gd}$ and ${ }^{192} \mathrm{Hg}$.

into the HF calculations constraining merely the expectation values of the $\hat{K}_{1}$ isoscalar operator.

The angular velocities within the three studied approaches, namely $\mathrm{HF}$, HFB and $\mathrm{HF}+\mathrm{V}$, are plotted in Fig. 4 against the angular momentum. It is seen that the $\mathrm{HFB}$ and $\mathrm{HF}+\mathrm{V}$ values fully overlap:

$$
\Omega_{H F B} \simeq \Omega_{H F+V} .
$$

It is worth noting that these two angular velocities are greater than $\Omega_{H F}$. This is not surprising, since the counter-rotating collective effect of pairing has to be compensated in HFB calculations at a given angular momentum by an increase of the angular velocity. However, it is striking to see that the identity of the two momenta of the current-distribution, namely $I$ and $\left\langle\hat{K}_{1}\right\rangle$ in HFB and $\mathrm{HF}+\mathrm{V}$ calculations, leads to identical rotational angular velocities $\Omega$. It proves indeed, more quantitatively than the plots of Fig. 3, that the current patterns in the two approaches are similar everywhere, which could hardly be demonstrated by merely looking at integrated quantities.

The identity of the HFB and $\mathrm{HF}+\mathrm{V}$ angular velocities seen in Fig. 4 yields an identity of the kinetic moments of inertia clearly demonstrated in eq. (6). As for the $\mathrm{HF}+\mathrm{V}$ dynamical moments of inertia, even though they do not fully coincide with their HFB counterparts, they are indeed very close and even reproduce better the experimental data. In this nuclei, the $\mathrm{HF}+\mathrm{V}$ and $\mathrm{HFB}$ formalisms yield solutions whose axial quadrupole moments remain constant over the entire band (differing by less than 2 percent around $Q_{20} \simeq 3900 \mathrm{fm}^{2}$ ). Hence the equivalence of the dynamical moments of inertia in the two formalisms is clearly free from any deformation effect and reveals similar superfluid properties.

\section{B. The yrast superdeformed band of ${ }^{192} \mathrm{Hg}$}

We have used also the HF, HFB+LN and $\mathrm{HF}+\mathrm{V}$ formalisms in order to describe the yrast superdeformed band of ${ }^{192} \mathrm{Hg}$ and compare their results with the available experimental data [25].

As already noted by Gall et al. [26] the very low amount of pairing correlations attained above $I \simeq 30 \hbar$ (as exemplified by the pairing energies for both neutrons and protons in the corresponding lower part of Fig. 2) makes it necessary to correct for the particle number symmetry breaking. This has been performed upon using the approximate Lipkin-Nogami particle number projection on top of the HFB calculation which has been found necessary to approach a proper description of the rotational band of this nuclei and avoid spurious drops in the moments of inertia due to sudden pairing-energy disappearance both for neutrons and protons. Whereas the LN prescription is known to be efficient in restoring some pairing correlations in weak-pairing regime as would be the case in HFB calculation for these nuclear states, the question of knowing whether or not it provides the correct amount of pairing correlations is still controversial (see, e.g., the discussion of Peru [27]). A more exact treatment of pairing correlations could be obtained for instance by generalizing to time-reversal symmetry breaking systems the method of Pillet et al. $[28,29]$ which explicitly con- 

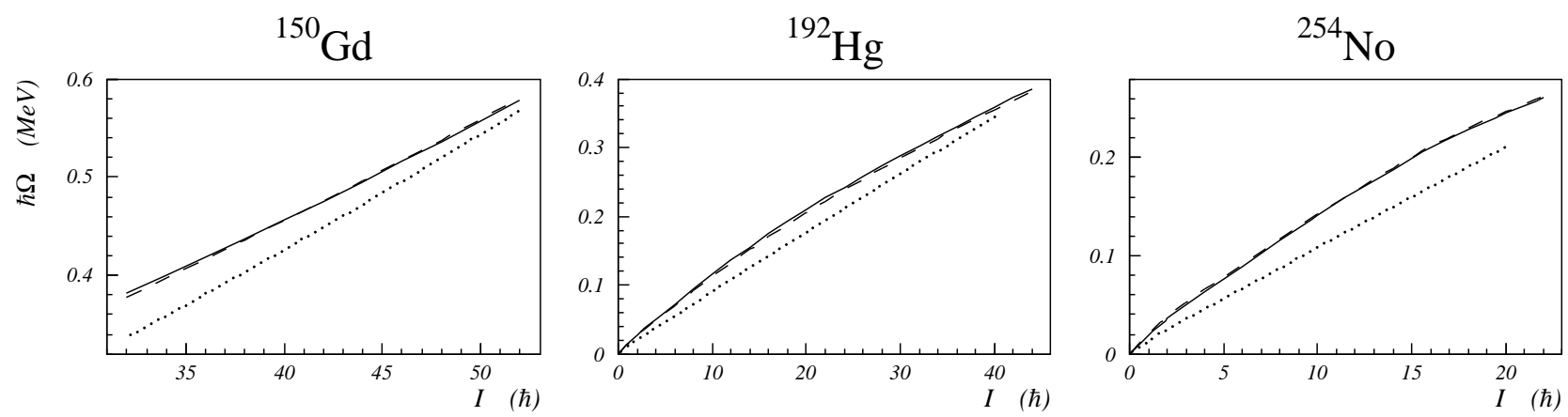

FIG. 4. Angular velocity $\Omega$ (in $\mathrm{MeV}$ ) as a function of the nuclear spin $I$ (in $\hbar$ units) for the three considered nuclei. The conventions in use are the following: $\mathrm{HF}$ value (dotted line), $\mathrm{HFB}$ value (full line), and $\mathrm{HF}+\mathrm{V}$ value (dashed line). The Lipkin-Nogami correction has been applied for ${ }^{150} \mathrm{Gd}$ and ${ }^{192} \mathrm{Hg}$.

serves the particle number.

The ${ }^{192} \mathrm{Hg}$ dynamical moments of inertia are plotted in the upper panel of Fig. 1 within the three mentioned formalisms together with their experimental counterparts. As was already the case for ${ }^{150} \mathrm{Gd}$, the $\mathrm{HF}$ calculations fail to reproduce the experimental values as well as the behavior of this moment as a function of the angular velocity since it remains almost constant over the whole rotational band. By adding pairing correlations within the HFB+LN formalism, it is possible to reproduce the experimental values at low spins. However, using a simple seniority force as done here, one can only reproduce the increasing trend of the experimental moment of inertia and our theoretical value becomes to high at the upper bound of the rotational band. In view of some residual instabilities of the solution due to the presence of single-particle states in the vicinity of the cutoff energy defining the configuration space where pairing correlations are treated, we have introduced for this nucleus a cutoff factor of the Fermi-function type.

Similarly to what we have done for the ${ }^{150} \mathrm{Gd}$ nucleus, we have attempted to model the collective effects of pairing correlations in this nucleus by performing $\mathrm{HF}+\mathrm{V}$ calculations. As seen in Fig. 1, the calculated $\mathrm{HF}+\mathrm{V}$ dynamical moments of inertia nicely match the HFB+LN ones, even better than for ${ }^{150} \mathrm{Gd}$. The axial deformation of the ${ }^{192} \mathrm{Hg}$ nuclear states in the rotational band calculated within these two formalisms differ by less than 3 percent with an axial quadrupole moment mean value of $Q_{20} \simeq 44$ barns.

In view of the rather low expectation values of the Kelvin circulation in the HF regime (see Fig. 2) its behavior as a function of the angular momentum in the HFB approach reflects mostly the interplay between the global rotation and the pairing correlations. One observes indeed a very interesting parabolic pattern. At low spins its low value is due to the fact that the intensity of the intrinsic currents is obviously related to the intensity of the global rotational currents which they are counteracting. On the other hand these intrinsic currents are, as we have assumed and demonstrated here, related with the intensity of pairing correlations. This balance explains the appearance of a maximum for $\left|\left\langle\hat{K}_{1}\right\rangle\right|$ at some "intermediate" spin value (around $I \simeq 20 \hbar$ ). From the plotted value of $\left\langle\hat{K}_{1}\right\rangle$ for both charge states in Fig. 2, one may infer that as it was the case for ${ }^{150} \mathrm{Gd}$, the pairing generated intrinsic currents are almost entirely of an isoscalar character.

The agreement between $\mathrm{HFB}$ and $\mathrm{HF}+\mathrm{V}$ dynamical moments of inertia clearly points into the direction of identical current patterns for both theoretical descriptions. This is indeed so, as shown in Fig. 3. Finally, one observes in Fig. 4 that the similarity of the kinetic moments of inertia in the two theoretical descriptions is reflected in the corresponding curves giving the spindependence of the angular velocity.

\section{The ground-state deformed band of ${ }^{254}$ No}

Recently, the existence of the rotational ground-state band of the heavy nucleus ${ }^{254}$ No has been assessed [30] by an observation of the $\gamma$-transition energies from spins $I=4 \hbar$ up to $I=20 \hbar$ in coincidence with the $\alpha$-decay chain of this nucleus. This high spin structure is the first one studied in $Z>100$ nuclei.

It has been shown by several authors $[14,31,32]$ with different effective forces and pairing parameterizations that the properties of this isotope could be reproduced within HFB-type formalisms. The calculations presented here have been performed in the spirit of Ref. [14], that is fixing the pairing-force parameterization in order to reproduce the first transition energies. As a result, it happens that higher transition energies are fairly well reproduced. This procedure has been found in [14] to be more successful within a pure HFB than a HFB+LN formalism. Thus we will discuss here in terms of intrinsic vortical currents the results of our HFB calculations.

The dynamical moments of inertia obtained within the three formalisms $\mathrm{HF}, \mathrm{HFB}$ and $\mathrm{HF}+\mathrm{V}$ are plotted in the corresponding upper panel of Fig. 1 as functions of the angular velocity. As was the case for the two previously 
studied nuclei, it is still impossible here to reproduce the experimental data with pure HF calculations. On the contrary, the results of our HFB calculations fit rather well in the experimental patterns, and the $\mathrm{HF}+\mathrm{V}$ results fully overlap the HFB ones. A slightly less good agreement between both theoretical estimates of $\mathfrak{J}^{(1)}$ among themselves and with their experimental counterpart is obtained as exhibited in Fig. 1.

In Fig. 2, the HF and HFB estimates of $\left\langle\hat{K}_{1}\right\rangle$ for the ${ }^{254}$ No neutron current distributions are seen to be very close. This is clearly related as exhibited in the lower part of this figure to the very small amount of pairing correlations for this charge state at spin less than $6 \hbar$ (whereas for higher spin values, there are no correlated solutions). As for the protons now, the shell effect contribution to $\left\langle\hat{K}_{1}\right\rangle$ (i.e., the HF value) is quite significant, actually of the same order of magnitude as the pairing contribution (i.e., the difference between the HFB and the $\mathrm{HF}$ value). As a very specific feature of the HF plus vorticity calculations in this nucleus (versus those above reported in ${ }^{150} \mathrm{Gd}$ and ${ }^{192} \mathrm{Hg}$ ) one notices that a purely isoscalar constraint on $\left\langle\hat{K}_{1}\right\rangle$ is not effective in reproducing the HFB results. Actually, whereas it is tempting to attribute such an isovector behavior for a $Z \sim 100$ nucleus (as opposed to $Z \sim 80$ or less nuclei) to the increasing relative importance of the Coulomb interaction (scaled as $Z^{2}$ ), the observed behavior of $\delta\left|\left\langle\hat{K}_{1}\right\rangle\right|$ (difference between $\mathrm{HFB}$ and $\mathrm{HF}+\mathrm{V}$ values of $\left.\left|\left\langle\hat{K}_{1}\right\rangle\right|\right)$ which is positive for protons and negative for neutrons is yet to be understood (and should be assessed by systematic calculations).

In Fig. 3 intrinsic currents corresponding to two very different rotational regimes $(I=2$ and $20 \hbar)$ are displayed for ${ }^{254}$ No. At low spins the neutron currents are small yet roughly consistent with a tangential pattern at the surface. This is due to the existence of a low pairing correlations regime. At high spin of course the HF and HFB neutron current patterns are coinciding and hardly tangential at the surface. This situation is quite at variance with what is obtained for the protons at large spins, where the prevalence of large pairing correlations entails the existence of rather well marked tangential counterrotating currents.

Finally, the behavior of the angular velocity of both $\mathrm{HFB}$ and $\mathrm{HF}+\mathrm{V}$ calculations on the one hand and of the HF calculations on the other hand, as a function of the total angular momentum is found quite similar here (see Fig. 4) to what was found before for ${ }^{150} \mathrm{Gd}$ and ${ }^{192} \mathrm{Hg}$.

\section{CONCLUSIONS}

In the above, we have examined in a Routhian approach, the equivalence of the dynamical effects yielded by pairing correlations à la Bogoliubov with those obtained when a specific ansatz for the collective flow is imposed onto a purely Hartree-Fock type of microscopic cal- culations. This has been achieved in rather different contexts as far as the nucleon number ( $A$ ranging from 150 to 250), the deformation (considering normally deformed bands as well as superdeformed bands), the range of spin values as well as the pairing correlation contents. In all cases however, we have found rather convincingly that constraining Hartree-Fock Routhian solutions to have the currents obtained by HFB Routhian calculations at the same spin values, was a sound substitute to these HFB calculations themselves.

For that purpose, the so-called Kelvin circulation operator has appeared as an efficient measure of the intrinsic currents. Since this operator is well suited to the collective dynamics dubbed as S-type ellipsoids by Chandrasekhar (implying a collective field which is linear in the coordinates), the actual HFB dynamical pattern should not deviate very much from this simple model pattern. That has been actually assessed by a mere inspection of current lines obtained in our HFB calculations in cases where at large enough spin values strong pairing correlations where present. Pairing correlations appear indeed as generating tangential counter-rotating currents in the intrinsic frame. This equivalence has been actually confirmed by a convergent array of evidences:

i) the similarity of dynamical moments of inertia,

ii) the congruence of kinetic moment of inertia, or equivalently (at the same value of the angular momentum) of the associated angular velocity,

iii) the correspondence between currents obtained in both approaches.

As for the currents, it has appeared that shell effects where visible in situations where either the collective intrinsic flow was too small (at low angular velocity for the global rotation) or when pairing correlations where vanishing. These shell effects generating density inhomogeneities are responsible for erratic current patterns (sometimes roughly similar to localized vortices) which are in general neither counter-rotating nor tangential.

As above discussed, upon increasing the spin value one experiences a competition between two processes. The first one is an increase of the intrinsic currents, a reaction effect which should be (and actually is, all things kept equal) increasing with the global rotation angular velocity. This type of currents decreases, as well known, the kinetic energy at a given angular velocity. The second one is the Mottelson-Valatin rotation-generated reduction of the pairing correlations on. The competition arises from the fact that this intrinsic flow should be decreasing with the decrease of the pairing correlations. One may thus easily understand the maximum obtained for the absolute value of the expectation value of the Kelvin circulation obtained for the ${ }^{192} \mathrm{Hg}$ nucleus at intermediate spin values.

In our calculations an isoscalar constraint on the Kelvin circulation operator has been used. Whereas it 
has been deemed to be adequate for the two lighter nuclei under study, this has been hardly the case for the heavier one. Confirming and, if so, understanding this $A$ dependence or more likely $Z$-dependence, would require a rather systematic study which has not been performed here.

The present study is now extended in two directions.

At high spins the decrease of pairing correlations has often been extrapolated towards the appearance of a phase transition (associated thus with a complete disappearance of these correlations). It is clearly not very appropriate to quantitatively assess this feature in traditional approaches because one reaches there a low pairing regime where the Bogoliubov particle number symmetrybreaking approach is not very adequate there as well known. We are currently undertaking [33] Routhian calculations which explicitly conserve the particle number according to the general lines proposed in Ref. [28].

Even though we are representing here in a rather simple way the dynamical effects of pairing correlations at finite angular momentum as obtained in a Bogoliubov approach, we are still needing such lengthy calculations to yield the correct amount of intrinsic currents (through the expectation value of the Kelvin circulation operator). The challenge is to try to correlate for a given nucleus (i.e., for given nucleonic numbers, given deformations and given single particle level densities near the chemical potential at zero spin or equivalently the amount of pairing correlations at zero spin) the Kelvin circulation expectation value as a function of the angular velocity of the global rotation. This is currently achieved through a simple model approach inspired from the Mottelson-Valatin rotation anti-pairing effect and will be presented soon in an other paper [34].

\section{ACKNOWLEDGMENTS}

Part of this work has been funded through an agreement (\# 12533) between the BAS (Bulgaria) and the CNRS (France) and another (\# 97-30) between the JINR (Russia) and the IN2P3/CNRS (France) which are gratefully acknowledged. One author (P. Q.) would like to thank the Theoretical Division of the LANL for the hospitality extended to him during his stay at Los Alamos. Two of the authors (H. L. and P. Q.) also wish to thank the INT (Seatle) for their kind invitation and the opportunity provided there to discuss some parts of this paper.

[1] D. Samsœn, P. Quentin and I. N. Mikhailov, Phys. Rev. C 60, 014301 (1999).

[2] A. Bohr and B. R. Mottelson, Mat. Fys. Medd. Dan. Vid. Selsk. 30, 1 (1955).
[3] S. D. Belyaev, Nucl. Phys. 24, 322 (1961).

[4] R. Y. Cusson, Nucl. Phys. A114, 289 (1968).

[5] I. N. Mikhailov, P. Quentin and D. Samsœn, Nucl. Phys. A627, 259 (1997).

[6] S. Chandrasekhar, Ellipsoidal Figures of Equilibrium (Dover, New York, 1987).

[7] I. N. Mikhailov, C. Briancon and P. Quentin, J. of Particles and Nuclei 27, 303 (1996).

[8] M. Durand, P. Schuck and J. Kunz, Nucl. Phys. A439, 263 (1985).

[9] J. Kunz and U. Mosel, Nucl. Phys. A323, 271 (1979).

[10] J. Fleckner, J. Kunz, U. Mosel and E. Wüst, Nucl. Phys. A339, 227 (1980).

[11] M. Radomski, Phys. Rev. C 14, 1704 (1976).

[12] G. Rosensteel, Phys. Rev. C 46, 1818 (1992).

[13] H. Laftchiev, Ph.D. thesis, Université Bordeaux-I and INRNE Sofia, 2001.

[14] H. Laftchiev, D. Samsœn, P. Quentin and J. Piperova, Eur. Phys. J A 12, 155 (2001).

[15] D. Samsœen, P. Quentin and J. Bartel, Nucl. Phys. A652, 34 (1999).

[16] Y. Lipkin, Phys. Rev. B 134, 313 (1964); Y. Lipkin and I. J. Zucker, Nucl. Phys 60, 203 (1964); Y. Lipkin, Phys. Lett. 156, 335 (1965);

J. F. Goodfellow and Y. Lipkin, Can. J. Phys. 44, 1321 (1966);

H. C. Pradhan, Y. Lipkin and J. Law, Nucl. Phys. A201, 357 (1973).

[17] P. Quentin, D. Samsœn and I. N. Mikhailov, in preparation.

[18] J. Bartel, P. Quentin, M. Brack, C. Guet and H.-B. Haakansson, Nucl. Phys. A386, 79 (1982).

[19] P. Fallon et al., Phys. Lett. 257B, 269 (1991).

[20] S. Erturk et al., Proceedings of the Second Balkan School on Nuclear Physics, 2000.

[21] P. Bonche, H. Flocard and P.-H. Heenen, Nucl. Phys. A598, 169 (1996).

[22] K. Bencheikh, P. Quentin and J. Bartel, Nucl. Phys. A571, 518 (1994).

[23] B. R. Mottelson and J. G. Valatin, Phys. Rev. Lett. 5, 511 (1960).

[24] C. Kittel, Quantum Theory of Solids (Wiley, New York, 1963).

[25] B. Gall et al., Z. Phys. A 347, 223 (1994).

[26] B. Gall, P. Bonche, J. Dobaczewski, H. Flocard and P.-H. Heenen, Z. Phys. A 348, 183 (1994).

[27] S. Peru, Ph.D. thesis, Université Paris-XI, 1997.

[28] N. Pillet, P. Quentin and J. Libert, Nucl. Phys. A697, 141 (2002).

[29] N. Pillet, Ph.D. thesis, Université Bordeaux-I, 2000.

[30] P. Reiter et al., Phys. Rev. Lett. 82, 509 (1999); M. Leino et al., Eur. Phys. J. A 6, 63 (1999); P. Reiter et al., Phys. Rev. Lett. 84, 3542 (2000).

[31] T. Duguet, P. Bonche and P.-H. Heenen, Nucl. Phys. A679, 427 (2001).

[32] J. L. Egido and L. M. Robledo, Phys. Rev. Lett. 85, 1198 (2000).

[33] H. Laftchiev, J. Libert and P. Quentin, in preparation.

[34] H. Laftchiev, D. Samsoen, P. Quentin and I. N. Mikhailov, in preparation. 